Aus Dr. Max Jeseph's Poliklinik für Hautkrankheiten in Berlin.

\title{
Über ein von der Nase ausgehendes Syphiloma hypertrophicum diffusum faciei (Elephantiasis luetica).
}

Von

\section{Dr. Gottfried Trantmann}

aus Minchen.

Außer der Filaria sanguinis, welche in überseeischen und tropischen Ländern die Elephantiasis bedingt, veranlassen verschiedene Krankheiten eine Volumszunahme irgend eines Körperteils, die man wegen ihrer Ähnlichkeit mit ersterer ebenso benannt hat.

Auch die Lues tritt unter ihren verschiedenen Formen, und zwar nicht selten, als Elephantiasis auf.

Es ist das Verdienst $\mathrm{Mr}$ a č eks, dieselbe in einer längeren Abhandlung (die Elephantiasis infolge ron Syphilis und das Syphiloma hypertrophicum diffusum, Wiener Klinische Wochenschrift 1888, Nr. 12 mit 15) kritisch beleuchtet und auf ihre verschiedenen Arten aufmerksam gemacht zu haben.

Mraček unterscheidet zwei Formen: 1. eine, welche auf der Bindegewebshypertrophie beruht und durch die Syphilisprodukte nur eingeleitet wird; und 2. eine, die ihrer äußeren Form nach mitunter an die Elephantiasis erinnert, im wesentlichen aber directes Syphilisprodukt ist, das durch eine umfangreiche syphilitische Infiltration entsteht.

Zur ersteren gehört das Oedema indurativum, welches durch eine von Sklerosen und Papeln in der Umgebung veranlaßte Erkrankung der Lymphgefäße und Lymphdrüsen entsteht und eine oft beträchtliche Hypertrophie des subkutanen Bindegewebes und infolge dessen eine oft erhebliche Mißgestaltung des betroffenen Körperteiles, besonders der äußeren Genitalien, darstellt, analog also der Elephantiasis, die auf Lymphstauungen beruht.

$\mathrm{Höh}$ er e Grad e dieser elephantiastischen Bindegewebswucherungen, mit Vorliebe am Unterschenkel, führen neben oberflächlichen Gummi

Areh. f. Dermat. u. Syph. Bd. LXIII. 
ganz besonders die tiefen, im Unterhautzellgewebe befindlichen herbei, wie wir es auch von parenchymatösen Organen z. B. der Leber wissen, wo es oft zur diffusen Bindegewebswucherung kommt.

Durch Zerstörung der Blut- und Lymphgefäße durch Olzerationen oder Verlegung ersterer durch essentielle Vernarbungen werden die Zirkulationsverhältnisse sehr schlechte und die Bindegewebswucherung nimmt in erhöhtem Maße zu.

Bei periostalen und ostalen syphilitischen Affektionen entstehen die gleichen Verhältnisse. Mraček hat bei Nekrose der Nasenknochen und des Oberkiefers, besonders des unteren Orbitalrandes wiederholt eine bleibende Anschwellung des Unterhautzellgewebes beobachtet.

Diese Art der Elephantiasis ist also nur eine Begleit- oder Folgeerscheinung der Syphilis, und es ist eine vollständige Rückbildung durch eine spezifische Behandlung nicht möglich. Durch diese heilen nur die sie bedingenden syphilitischen Affektionen. Die Therapie muß also im wesentlichen eine chirurgische sein.

Die z weit e Form ist etwas ganz anderes. Sie kommt ausschließlich in den gummösen Stadien der Lues vor, und zwar besteht sie nicht in einem Aggregat von enganeinander gedrängten, einzelnen Gummis, die auf diese Weise als ein gleichmäßjges Infiltrat imponieren können, sondern sie stellt eine mebrweniger diffuse, gummöse Infiltration des Gewebes dar, ist also keine Neben- oder Folgeerscheinung der Syphilis, sondern eine besondere Form derselben.

Für diese elephantiastischen Infiltrationszustände hat $\mathrm{B}$ i don (Thèse de Paris 1886, c. b. Mraček) den Namen Syphiloma hyper. trophicum diffusum eingeführt.

Fournier (die gummösen Syphilide, Indépendance méd. 1899, Nr. 49) sagt, daß zuweilen sich zur tertiären, ulcerösen Form der Lues eine Hypertrophie neben elephantiastischer VergröBerung der Umgebung gesellt und daß dieselbe (Syphilom hypertrophique diffus oder Syphilide léontiasique) mit Vorliebe Unterlippe, Nase, Ohr befällt.

Die bis jetzt publizierten Fälle waren auch vorwiegend im Gesichte, meistens an den Lippen, Wangen, an der Nase lokalisiert und auch an der Zunge, und zwar kombiniert oder a uch als alleinige Erkrankung, welcher Zeissl den Namen Glossitis indurativa diff us a gegeben hat.

Den Hinweis auf die oben zitierte Abhandlung Mračeks möchte ich deshalb stark betonen, weil ich in den gangbaren Lehrbüchern diese Form der Lues nicht in der gleichen deutlichen Art beschrieben finde. Die sechs Fälle Mračeks sind in kurzem folgende:

1. Fall: Infektion vor 6 Jahren, Student, $27 \mathrm{~J}$., Inunktionskur und Zittmann vor 4 J. Diffuse Infiltration beider Nasenflügel und des Septums. Derbe Infiltration und Hypertrophie beider Mundlippen, namentlich 
der oberen mit Rhagadenbildung an den Mundwinkeln. Beginn der diffiusen' Infiltration vor $1 \frac{1}{2}$ Jahren. Rü ckbildung der Form naeh s0täg. Behandlung (lokal Carbol und graues Pflaster, JK innerlich, 19 Einreib. mit Unga. ein.). Partielle Rezidive an der Oberlippe mit Knotenbildung und neve Anschwell. an den Genitalien. Besserung.

2. Fall: Infektion anamnestisch nicht nachweisbar, Bauernsohn, 16 J. Diffuses hypertrophisehes Syphilom der ganzen Oberlippe, der knorpeligen Nase mit Perforation des Septum. Daner der Erkr. 4 Jahre. Rückbildung nach einer 239 'Tage währenderi energ. Beh. (Jodoformpillen, Jodkali mit Ferrum, 60 Inunktionen à 5.0 Ungu. cin.).

3. Fall: Infektion vor $14 \mathrm{~J}$, kurze Zeit Hg-Pillen, dann Kaltwas serkur. Fabrikbesitzer, 34 J. Diffuses hypertroph. Syphilom der linken Hälfte der Unterlippe. Infiltration der Zungenoberfläche. Heilung nach 4 monatl. Behandlung (lokal graues Pflaster, Bepinsl. mit Jodlös., intern Zittmann; Inunktionen).

4. Fall: Infektion vor $23 \mathrm{~J}$, , ohne systemat. antiluet. Beh., Beamter, 43 J. Diff. hypertr. Syphylom der Oberlippe, syph. Knotenbildung in der Zunge, oberfl. Hautgummi an der Stirne. Schwell. des Sept. der Nase mit Perforation desselben. Narben nach Gumm. der Haut, der Nase und der Wade. Heilung nach 1/2 jähr. energ. Beh. (Zittmann, Inunktionen).

5. Fall: Vor 9-10 J. Genitalgeschwür. Syph. Infiltration des Periostes und des Zellgewebes der Orbitalhöhle. Übergreifen des Proj. auf den obliqu. inf. und Abducens, Weiterschreiten desselben im subkutan. Zellgewebe der Lider, Wange, ferner im Perioste und Zellgewebe über dem Jochbogen. Entwicklung dieser Zustände im Verlauf von 6 Jahren. Rückbildung, wenn auch hartnäckig, nach wiederbolten antiluet. Kuren.

6. Fall: Pat. 40 J., Beginn der Erkr. vor 5/4 J. Makroglossie infolge einer diffusen, syph. Infiltration. Rü ckbildung nach 20 täg. B eb. (Zittmann, Jodbepinsl). Nach $1 / 4$ J. Zunahme der Infiltr. Sublimatinjektionen. Neuerliche Besserung.

Im Jahre 1891 demonstriert Mraček einen weiteren Fall ron Syphiloma hypertrophicum diffusum. (Wiener med. Doktorenkollegium, Sitz. 7./XII. 1901): Luet. Infektion nicht nachweisbar. Geschw. im Gesicht, an den Augen über die Nase gehend. Nach Inzision stets wachsendes Geschwür. Heilung durch Hg und JK.

Der von Thimm (deutsche med. Wochenschr. Nr. 24, 1895) aus Dr. Max Josephs Poliklinik beschriebene Fall von syphilitischer Makrocheilie ist wohl auch zur Gruppe der diffusen, hypertrophisehen Syphilome zu rechnen. Es spricht hiefür die gleichmäßige Verdickung und Schwellung der Lippen und vor allem die Art der Wirkung der antiluetischen Therapie, welche bei sekundär durch einzelnø Gummi im Schleimdrüsengewebe verursachter Bindegewebswucherung doch auf die Verdickung ohne Einfluß geblieben wäre.

1897 demonstrierte Schwimmer (Ungar. dermat. und urolog. Ges. in Budapest, Sitz, 11./II. 1907.) einen Fall von Syphilis leontiasiformis: Auf der linken Gesichtshälfte, Stirne und behaartem Kopfe thaler- bis kindshandtellergroße Infiltrate, die rotbräunlich vom Niveau der Haut emporragen. Am Kinn links mehrere erbsen- bis haselnuGgroße drüsige Gebilde, rechts, am Naseneingang und Nasenrücken mehrere Knoten. schleimhaut unverändert. 
Ferner hat $L_{1}$ udwig $0 \mathrm{ulmann}$ (Beitrag zur Kenntnis der Glossitis luetica, Inaug.-Diss. Würzburg, 1897) 10 Fälle aus der Literatur und einen eigenen Fall von Zungenlues mitgeteilt, von denen 5 in die genannte Syphilomgruppe einzubeziehen sind ( 3 gebessert, 2 geheilt).

1898 beschreibt Thevenin (Journ. des mal. cut. et syph.) drei Fälle von tertiärem Zungen-Lippensyphilom. In einem von diesem war die ganze Unterlippe und zum Teil Oberlippe infiltrirt; an beiden Commissuren bestanden schmerzende und nässende Ulzerationen.

In der Literatur findet man noch mehrere Fälle, die als diffuse bypertrophische Syphilome gedeutet werden könnten.

Z. B. Allen (162. Sitz. d. New-Yorker derm. Ges. 23./III. 1886): Demonstration eines wucherenden Syphilodermas des Gesichtes eines $40 \mathrm{j}$. Farbigen, bei dem blumenkohlartige Entwicklung der Nase mit Schrunden und Ulzerationen festgestellt wurden, ferner Eichhorst (Virchows Archiv, Bd. 131, Heft 3) Elephantiasis syphilitica der Lippen, bei welcher die Schwellung und Wulstung der ietzteren vor 1 Jahre begannen und dieselben dann als 2 Daumen dicke, schwammige (!), rote Polster rüsselartig hervorsprangen. Schmierkur war hier erfolglos.

Jedoch ist bei denselben nicht mit Sicherheit derNachweis der dif $\mathrm{f} \mathrm{u} \mathrm{sen}$ Infiltration zu erbringen, und es liegt die Annahme nahe, daß es sich in diesen Fällen doch vielleicht nur um ein Konglomerat eng neben einander gesetzter Gummiknoten oder um Gummi mit gleichzeitiger oder nachgefolgter Bindegewebswucherung gehandelt hat. Für letzteres spricht jedenfalls das absolute Versagen einer antiluetischen Behandlung.

Durch die Liebenswürdigkeit des Herrn Dr. Max Joseph, für welche ich ihm an dieser Stelle meinen verbindlichsten Dank sage, ist es mir möglich, aus seiner dermatologischen Poliklinik einen Fall zu beschreiben, der nach seinem

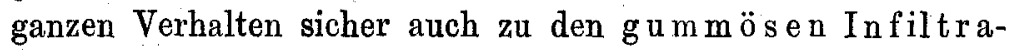
tionszuständen, zu dem Syphilomahypertrophium diffusum, als eine Form der syphilitischen Elephantiasis, gerechnet werden muß. Der Fall ist folgender:

Elephantiasis s. Syphiloma diffusum faciei von der Nase ausgehend. Diffuse gummöse Infiltration und Hypertrophie der äußeren Nase und vorderen Nasenschleimhaut, sowie beider Wangen. Derbe Infiltration und Hypertrophie der Oberlippe. Knoten, schmerzhafte Rhagaden und Ulzerationen, besonders an der Oberlippe. Infektion nicht nachgewiesen. Rückbildung nach 50tägiger Behandlung mit JK.

R. A., Tischler, 77 Jahre alt, während seiner Militärzeit 1845 genitalkrank, nach seiner Angabe nur Gonorrhoe. 1847 wurde er von einem Pferde auf den Kopf geschlagen, will mit dem Gesicht auf das Stallpfiaster gefallen sein and im Anschluß daran eine erhebliche Ver- 
letzung der äuBeren Nase davon getragen haben, die chirurgisch behandelt wurde.

Seine jetzige Nasenzrankheit soll vor $13 \mathrm{Jahren}$ mit Rötung: und Schwellung begonnen haben und hat sich in der Folgezeit auf die angrenzenden Wangen ausgedehnt. Er wurde von verschiedenen Seiten mit Salben und Umschlägen behandelt. Vor $1 \frac{1}{2}$ Jahren beobachtete er, hauptsächlich rechts, Abnahme der Gehörschärfe, die fernerhin, aber nicht sebr erheblich, zunahm. Kein Ohrensausen. Vor $1 / 2 \mathrm{~J}$ a hre bemerkte er auch die Oberlippe befallen, die Bildung neuer Knoten und das Auftreten von Ulzerationen an der Nase, an den Wangen und an der Oberlippe, welche zumeist schmerzhaft waren. Zugleich machten sich subjektive Beschwerden als spannendes Gefühl in den ergriffenen Teilen, Bewegungsbeschränkung der Lippen, erschwertes Sprechen und Kauen geltend. Das Gesicht bekam einen starren Ausdruck, die Haut eine lividrote Färbung uud gleichmäßige Härte und Verdickung, so daß es eine starke Mißgestaltung zeigte. In der Zwischenzeit soll Pat. an einem Rhinophym behandelt, scarifiziert und auch galvanokauterisiert worden sein.

Status. Pat, ist ein breitschulteriger, kräftiger und trotz seiner 77 Jahre keineswegs greisenhafter Mann. Das Gesicht masht einen starren, leontiasisähnlichen Eindruck.

Der größte Teil desselben ist von einem Krankheitsprozeß eingenommen, der sich von der Verbindungslinie beider Augenbrauen nach abwärts über die ganze Nase erstreckt und beiderseits kontinuierlich auf die Wangen übergeht, wo er mit einer von den inneren Augenwinkeln bis zur Unterkiefermitte gehenden Linie ziemlich scharf gegen die gesunde Haut abschneidet. Nach unten von der Nase setzt sich der Prozeß auf die gesamte Oberljppe fort und greift beiderseits in die Wangenaffektion über.

Die Farbe dieser befallenen Partien ist lividrot, zum Teil von einem eigenen Glanz, aber nirgends ist eine merkbare venöse Stauung oder Gefäßneubildung vorhanden.

An verschiedenen Stellen sieht man zirka erbsengro Be Erhabenheiten, auf der Haut der knorpeligen Nase, an den Nasolabialfalten und den beiden Wangen unregelmälige und runde Ulzerationen bis Linsengröße, auf der Oberlippe ein ziemlich tiefes zirka $2 \mathrm{~cm}$ langes, $1 / 2 \mathrm{~cm}$ breites Geschwür neben kleineren $\mathrm{Substanzverlusten.}$

Auf der Nasenbeinhant befindet sich eine breite, weiche $\mathrm{N}$ a r be, kleinere rundliche und streifenförmige Narben sind vereinzelt an anderen Stellen.

Bei der Palpation sind die einzelnen Knoten hart, die Geschwüre schmerzhaft, das ganze befallene Gebiet ist von s ehr d erber Kon sistenz, plattenförmig infiltriert und verdickt. Besonders die Oberlippe zeichnet sich durch Härte und Volumszunahme aus und steht wulstförmig nach vorne. 
Das rechte $\mathrm{Na}$ a e $\mathrm{n}$ lo $\mathrm{ch}$ ist dorch die dasselbe umgebeode Infiltration verengt und nur für Bleistiftdicke durchgängig, das linke ebenfalls rerengt, aber in geringerem Grade. Die schwer sichtbare Nasenschleimhaut ist verdickt und macht eine rhinoskopische Untersuchung nicht möglich. Die Nasenatmung ist kaum behindert.

Die $\mathrm{Zunge}$ und $\mathrm{Mundh}$ hlle ist frei von Erscheinungen, erstere aber schwer beweglich unter dem Einfluß der äußeren Erkrankung. Kaubewegungen und Sprechen erschwert aus demselben Grund. Bei der Ohrenuntersuchung ergibt der otoskopische Befund nicht anormales. Bei der funktionellen Prüfung werden bei Konversationssprache rechts die Zahl 8 in $2 \mathrm{~m}$, die Zahl 3 , Otto, Friedrich in $1 \mathrm{~m}$ Entfernnng, bei Flüstersprache die Zahl 8 in $0.5 \mathrm{~m}$, die Zahlen 3, 16,20 in $025 \mathrm{~m}$ Entfernung gehört; links Konv. 8, 14, 20, Otto in $3 \mathrm{~m}$, Flüst. 8,14 in $0.5 \mathrm{~m}, 3,5,16$ in $0.25 \mathrm{~m}$. Weber'scher Versuch: wird nicht lateralisiert, Rinne: normal. Tiefe Töne: beiderseits mäßig herabgesetzt; ho he Töne (C 4): beiderseits, besonders rechts, nahezu anfgehoben. Dieser Befund macht den Erkrankungssitz im in neren $\mathrm{Ohr}$ wahrscheinlich.

Therapie: JK und Umschläge mit essigs. Tonerde auf die erkrankten Partien des Gesichts.

Patient gibt nur eine Gonorrboe zu und stellt eine luetische Infection in Abrede. $\mathbf{E r}$ ist an einem Rhinophym behandelt worden, und zwar angeblich von einer Seite, bei welcher die Diagnose nicht in Zweifel gezogen werden kann. Das liegt aber schon länger zurück.

Der erste Anblick des Prozesses, der sich in der Hauptsache auf Nasenrücken, Nasenflügel, angrenzenden Wangen, aber auch auf die Oberlippe erstreckt, könnte einen auch in der Annahme bestärken, daß es sich auch jetzt noch ausschließlich um ein Rhinophym handelt. Die Lokalisation, die zum Teil lividrote Farbe, die unregelmäßigen, knotigen Erhabenheiten sprechen dafür. Auch neugebildetes Bindegewebe, wie es beim Rhinophym vorkommt, macht mitunter den Eindruck eines diffusen Prozesses.

Trotz der nicht zugegebenen luetischen Infektion mußte man aber doch bei näherer Untersuchung zu dem Schlusse kommen, daß man es mit luetischen Erscheinungen zu tun hat. Auch andere Krankheiten waren auszuschließen.

Vor allem ist es die Kon is te nz, die sich beim Befühlen als eine harte erweist, und die der Patient selbst empfindet, da sie ihm Funktionsstörungen beim Naseschnauben, beim Kauen der Speisen, beim Sprechen, ferner ein Spannungsgefühl 
in der Haut verursacht, während beim Rhinophym das befallene Gebiet im ganzen weich bleibt.

Die einzelnen Knoten auf harter Grundlage sind ebenfalls $d e r b$ und zeigen nicht einen Gefäßreichtum, wie beim Rhinophym. Selbst weiche Gummiknoten sind anders.

Die lividrote Farbe ist nicht an eine venöse Stase gebunden, sie ist diffus und läßt in ihrem Bereich keine Gefäßbäumchen erkennen. Akneknoten oder Pusteln, Komedonen fehlen.

Als fernerer Punkt dagegen spricht das Übergreifen des Prozesses a út die Oberlippe, was beim Rhinophym nicht vorkommt und als entscheidendes Moment die Rhagaden und Ulzerationen an der Nase, an den Wangen und vor allem auf der Oberlippe, wo sie in großer Ausdehnung vorhanden sind.

Abgesehen von der großen flächenhaften Narbe auf der Nasenbeinhaut, die nach der Anamnese auf einen Fall auf das Gesicht zurückgeführt werden muß, zeigt der Prozess an verschiedenen Teilen mehrweniger große zirkumskripte und streifenförmige Narben, die aber zum großen Teile doch auf Rechnung der früher stattgehabten Scarificationen und Galvanokauterisationen gesetzt werden müssen, obgleich nicht von der Hand zu weisen ist, daß einzelne von ihnen spontane Heilungsresultate bestandener Geschwüre sein können. Diagnostisch konnten sie aber nicht verwertet werden.

So wurde denn die Diagnose Elephantiasis luetica gestellt und die Behandlung mit Jodkali intern und lokal mit Umschlägen mit essigsaurer Tonerde eingeleitet. Von einer energischen Inunktionskur wurde bei dem hohen Alter des Patienten ( 77 J.) umsomehr Abstand genommen, als die Erscheinungen keine lebensgefährdende waren.

Unter dieser Therapie gingen nun die Erscheinungen im Verlaufe von 50 Tagen wesentlich zurück. Die befallenen Teile verloren zunächst ihre harte Konsistenz und wurden weich, wodurch die Funktionsbehinderungen, in erster Linie das erschwerte Kanen, ferner die Beweglichkeitsbeschränkung der Lippen, die undeutliche Sprache und das. Spannungsgefühl beseitigt wurden. Die knotigen Erhabenheiten wurden kleiner, flacher and auf der nun weicher und glatter gewordenen Unterlage ebenfalls weich. Die schmerzhaften Rhagaden 
und die Ulzerationen vernarbten, die Farbe hat an Intensität verloren und ist heller geworden. Die Nasenlöcher sind reweitert, die noch verdickte Schleimhaut weich. In der Nasenhöhle sonst nichts abnormes.

Das ganze Bild ist ein anderes geworden; es zeigt sich im Stadium des fortschreitenden Zurückgehens, der Heilung. Die antiluetische Behandlung hatte also Erfolg.

Nachdem nun auf diese Weise die Diagnose Lues gesichert erschien, handelte es sich noch um Feststellung der syphilitischen Form. Hiebei folge ich den differential-diagnostischen Leitsätzen Mračeks.

Ein induratives Ödem war auszuschließen, anamnestisch sowohl, als aus dem Mangel der Anschwellung der benachbarten Lymphdrüsengruppen. Außerdem war der ganze Prozess viel zu diffus.

Dagege $\mathbf{n}$ kam in Frage, ob die elephantiastische Mißgestaltung auf einen Convolut aneinander gereihter und zum Teil einzeln stehender Gummiknoten beruhte, oder jene syphilitische Elephantiasis darstellte, die eine Sekundärerscheinung - eine Bindegewebswucherung - bei vorhandenem Gummi ist, oder ob man es mit einer gummösen Gewebsinfiltration, mit einem Syphiloma hypertrophicum diffusum, zu tun hatte.

Diagnostisch bieten diese drei Arten manchmal wenig Unterschiedliches, indem sie alle das Bild einer mehr oder weniger gleichmäßigen Infiltration zeigen können. Die differentiellen Merkmale werden hingegen erst bei weiterer Beobachtung des Falles und zwar in seinem speziellen Verhalten gegen die wirksame antiluetische Therapie deutlich. So auch in dem beschriebenen Falle.

Bei Vorhandensein des ersten Zustandes hätten wir Geschwüre vor uns, die, wie Mraček sagt, grubig vertieft sind und den einzelnen Centren der erweichten Knoten entsprechen. Durch die Behandlung würde sich die ganze Schwellungspartie bei der Involution wiederum in eine Summe einzelner sicht- oder fühlbarer Knoten aufgelöst haben.

Im zweiten Falle wären durch das JK wohl die Gummi zurückgegangen, aber die starre Bindegewebswucherung wäre unbeeinflusst geblieben und es hätte sich ein Zustand von Furchen oder Vertiefungen als Heilungsresultat der ersteren 
und von Leisten oder Höokern als bleibender Zustand der letzteren herausgebildet. Man darf immerhin nicht vergessen, da:3 durch teilweise spontane Atrophie der gummösen Infiltration auch bei derdritten Art, dem diffusen hypertrophischen Syphilom, im Verlaufe sehr langen Bestehens, nicht aber während der Therapie, ein ähnliches Bild entstehen könnte. Ein solches war aber in unserem Falle weder vor, noch während der Behandlung in diesem Sinne vorhanden. Die verschiedenen, zum Teil auch unregelmäßigen Erhabenheiten - neben der gleichmäßigen Infiltration - finden wir auch beim Syphiloma hypertrophicum diffusum. Wir wissen (Mraček), da $B$ die Haut über diesen meistens im subkutanen Zellgewebe entstandenen Infiltraten anfangs kaum verändert, nach längerem Bestande lividrot geworden ist, bei gesteigertem Fortschreiten der Erkrankung papilläre Exkreszenzen entstehen. Auch können typische Gummi voraus und nebenher gehen. Damit kann man also wohl in unserem Falle die weniger einzelnen Erhabenheiten erklären.

Bei unserem Patienten hatten wir in der Hauptsache eine gleichmäßige, starre und harte, eine gummöse Infiltration und Hypertrophie der erkrankten Partien vor uns.

Was die Ges chwüre betrifft, so waren dieselben keine gummösen Zerfallsprodukte. Vor der Behandlung waren dieselben seichte, längliche, unregelmäßige Substanzverluste, auch tiefere Rhagaden, jedoch nicht in dem Grade, daß damit eine Infiltratseinschmelzung eingeleitet wurde.

Diese Ulzerationen waren auch an Stellen, die einer von außen gesetzten Läsion, z. B. an der Nasolabialfalte und einer Zerrung, z. B. an den Mundcommissuren zugänglich waren. Diese Geschwüre entstehen auch durch anämische Nekrose, wenn durch die gummöse Infiltration daselbst den Gefässen die Ernährungsmöglichkeit abgeschnitten ist, sei es, daß sie zerstört oder verlegt sind. Diese Art von Geschwüren können zwar auch beim Gummi vorkommen, aber ausnahmsweise, dagegen beim diffusen, hypertrophischen Syphilom in der Regel. (Virchow, Geschwülste, pag. 402, Mraček 1. c.)

Die Ausbreitung des Prozesses fand von der Nase aus, als Zentrum peripherisch auf die Wangen und die Ober- 
lippe statt, wie es bei der letztgenannten Form der Fall zu sein pflegt.

Dementsprechend war auch die äußere Mißgestaltung eine elephantiastische, leontiasisartige und hiedurch die oben genannten Störungen verursacht.

Auf Grund dieses Tatbestandes scheint es berechtigt, in vorliegendem Falle die Diagnose auf Syphiloma hypertrophicum diffusum, als einer besonderen Art der Elephantiasis syphilitica zu stellen.

Die Involution der gummösen Infiltrate ist eine sehr langsame, und um sie vollständig zum Schwinden zu bringen, ist bei der Hartnäckigkeit des Leidens eine langdauernde, antiluetische Behandlung notwendig. Wir sehen jedoch aus den oben angeführten Fällen $\mathrm{Mračeks,} \mathrm{daß} \mathrm{schon} \mathrm{nach} \mathrm{ver-}$ hältnismäßig kurzer Zeit (20 und 30 Tage) eine Rückbildung zu konstatieren ist, und diese ist doch das Anfangsstadium der Heilung. Auch in unserem Falle ist nach einer Behandlung von 50 Tagen eine völlige Heilung noch nicht erreicht, aber eine ganz wesentliche Besserung eingetreten von der Art, wie wir sie oben geschildert haben.

Möglich ist es ferner auch, daß die durch die Ohraffektion bedingte Gehörstörung im Zusammenhang mit Lues steht. Eine merkliche Besserung derselben hat sich unter der JK-Behandlung bis jetzt nicht gezeigt, was aber nicht dagegen spräche.

Hinsichtlich der Beziehung des Rhinophyma zur Syphilis, speziell zur gummösen Infiltration, läßt es sich wohl kaum entscheiden, ob die hypertrophischen Talgdrüsen oder das gewucherte Bindegewebe gummös entartet werden, und es so zur Infiltration ganzer Gewebe kommen kann, oder ob hier der locus minoris resistentiae (Rhinophym und früheres Trauma) eine Rolle spielt. 